\title{
Evidence for the effects of decommissioning man-made structures on marine ecosystems globally: a systematic map protocol
}

\author{
Anaëlle J. Lemasson ${ }^{1 *}$ (D) Antony M. Knights ${ }^{1}$, Murray Thompson ${ }^{2}$, Gennadi Lessin ${ }^{3}$, Nicola Beaumont ${ }^{3}$, \\ Christine Pascoe ${ }^{3}$, Ana M. Queirós ${ }^{3}$, Louise McNeill ${ }^{3}$, Michaela Schratzberger ${ }^{2}$ and Paul J. Somerfield ${ }^{3}$
}

\begin{abstract}
Background: Numerous man-made structures (MMS) have been installed in various parts of the ocean (e.g. oil and gas structures, offshore wind installations). Many are now at, or nearing, the end of their intended life. Currently, we only have a limited understanding of decommissioning effects. In many locations, such as the North Sea, regulations restrict decommissioning options to complete removal, with little consideration of alternative management options might offer. To generate a reliable evidence-base to inform the decision-making processes pertaining to marine MMS management, we propose a wide-encompassing systematic map of published research on the ecosystem effects (including ecosystem services) of marine MMS while in place and following cessation of operations (i.e. including effects of alternative decommissioning options). This map is undertaken as part of the UKRI DREAMS project which aims to develop a system to show the relative effects of implementing different decommissioning strategies in the North Sea.
\end{abstract}

Method: For the purpose of this map, we will keep our focus global, in order to subsequently draw comparisons between marine regions. The proposed map will aim to answer the following two primary questions: 1. What published evidence exists for the effects of marine man-made structures while in place on the marine ecosystem? 2. What published evidence exists for the effects of the decommissioning of marine man-made structures on the marine ecosystem? The map will follow the Collaboration for Environmental Evidence Guidelines and Standards for Evidence Synthesis in Environmental Management. Searches will be run primarily in English in at least 13 databases and 4 websites. Returns will be screened at title/abstract level and at full-text against pre-defined criteria. Relevant meta-data will be extracted for each study included. Results will be used to build a database of evidence, which will be made freely available. This map, expected to be large, will improve our knowledge of the available evidence for the ecosystem effects of MMS in the global marine environment. It will subsequently inform the production of multiple systematic-reviews and meta-analyses.

Keywords: Oil and gas, Offshore wind, Marine renewable energy, North sea, Synthesis, Repurposing, Management, Ecology

*Correspondence: *anaelle.lemasson@plymouth.ac.uk

${ }^{1}$ School of Biological and Marine Sciences, University of Plymouth, Drake Circus, Plymouth PL4 8AA, UK

Full list of author information is available at the end of the article

\section{Background}

The marine environment has high biological productivity and high economic resource value, supporting intense human activity across multiple sectors including shipping, aggregate extraction and fisheries. In various parts of the world, many marine man-made structures (MMS)

c) The Author(s) 2021. This article is licensed under a Creative Commons Attribution 4.0 International License, which permits use, sharing, adaptation, distribution and reproduction in any medium or format, as long as you give appropriate credit to the original author(s) and the source, provide a link to the Creative Commons licence, and indicate if changes were made. The images or other third party material in this article are included in the article's Creative Commons licence, unless indicated otherwise in a credit line to the material. If material is not included in the article's Creative Commons licence and your intended use is not permitted by statutory regulation or exceeds the permitted use, you will need to obtain permission directly from the copyright holder. To view a copy of this licence, visit http://creativeco mmons.org/licenses/by/4.0/. The Creative Commons Public Domain Dedication waiver (http://creativecommons.org/publicdomain/ zero/1.0/) applies to the data made available in this article, unless otherwise stated in a credit line to the data. 
have been installed, for instance following the discovery of exploitable oil and gas, and marine constructions in general are now estimated to cover $32,000 \mathrm{~km}^{2}$ of our marine environment worldwide (1.5\% of global Exclusive Economic Zones; [1]). Over time the infrastructure installed will come to the end of its intended purpose and will require to be decommissioned. Hereafter, the term "decommission" will refer to the fate of a structure following cessation of operations and/or activities for which it was originally deployed, and will encompass all possible strategies and options from removal and disposal to repurposing and recycling (discussed further below).

Much of the already installed hard infrastructure is now at or nearing the end of its useful life, at least for the purposes for which it was installed. Decommissioning is taking place while simultaneously more and more structures are being installed (such as wind turbines). In the UK for instance, only $10 \%$ of the industry expenditure was on decommissioning in 2020, meaning that $90 \%$ is still spent on exploration, development, and operations [2]. Globally it is estimated that $>7500$ oil and gas platforms in the waters of 53 countries will become obsolete over the next several decades, and most will require complete removal under current regulations [3]. Owing to their size, weight, and in some cases age, the removal of platforms can be a complex engineering process and will require some of the heaviest lifting operations ever attempted at sea. While the oil and gas industry contributes a significant amount of money to national treasury through taxation, the global cost of removal has been estimated at US $\$ 210$ billion, with a substantial proportion of this cost provided to the industry through tax concessions [4]. While oil and gas activities ramp down (for instance in the North Sea), concern about climate change has led some countries to pledge to reduce their greenhouse gas emissions (e.g. UK national reduction target of $80 \%$ by 2050 compared to 1990 levels that is legally binding through the Climate Change Act). Strategies to achieve emission reductions generally include massive increases in off-shore wind generation capacity. Over the next 10 years, further rapid expansion of offshore windfarms (OWFs) is planned [5], leading to OWF hard infrastructure being installed over extensive areas of seabed. Yet, the eventual decommissioning of OWF (expected for over 1800 offshore wind turbines between 2020 and 2030, [6]; note that decommissioning of OWF has already occurred in some places, see [7]), will involve environmental considerations similar to those of oil and gas platforms [4].

What is lacking, particularly in regions such as the North Sea where the political and legislative context is complex (see below), is an assessment of the potential benefits, detriments, and trade-offs associated with different decommissioning strategies [8]. These are typically complex, as various stakeholders see things differently and have different end goals (see [9]). A strategy for decommissioning that benefits some stakeholders may be detrimental to others; e.g. a "Rigs-to-Reefs" strategy may benefit some conservation objectives by creating a de facto marine protected area, but may undermine fishermen who consequently cannot physically deploy certain gears there [10]. Therefore, there is an urgent need for evidence-based decision-making and management of MMS and their decommissioning, based on robust methodologies and on reliable and comprehensive evidence-bases, in order to provide the best possible advice to policy- and decision-makers, and optimize the trade-offs of the chosen management options [11, 12]. In places such as the North Sea, the legislation may need to be changed to enable the industry to adopt the chosen options.

Despite ongoing cessation of operations and decommissioning of marine MMS (e.g. oil and gas infrastructures, but also some OWFs), our understanding of the environmental effects of different decommissioning strategies is incomplete [4]. Past focus on a narrow set of criteria has limited evaluation of decommissioning effects [13], restricting decommissioning options in most regions, but particularly in the North Sea. There, the OSPAR Decision 98/3 on the Disposal of Disused Offshore Installations, which restricts decommissioning options in most cases to complete removal (except specific exemptions, see [13]), has meant that there has been little consideration of the potentially serious detriments of complete removal, nor the potential benefits of alternative decommissioning options (such as repurposing them; see below). At the same time, the OSPAR Agreement 1999/13 (now 2012/3) Guidelines on Artificial Reefs in relation to Living Marine Resources, which were also influenced by the controversy underpinning Decision 98/3, mean that oil and gas infrastructure cannot easily be repurposed either in situ or following relocation. Although OSPAR may appear restrictive, the legislation continues to be endorsed by the contracting parties and the EU commission, who ultimately seeks positive outcomes for all. Despite this, there is widespread acceptance that complete removal may not always be the most beneficial option [10, 14-16]. In some parts of the world, alternative strategies have been allowed, often with considerable success $[15,17]$. For instance, countries have allowed the relocation and/or alteration of oil and gas infrastructural components (generally jackets) to create artificial reefs (e.g. "Rigs-to-Reefs" programme in the USA, see $[17,18])$, or have repurposed them by converting them to other uses (e.g. hotel and dive resort, $\mathrm{CO}_{2}$ capture and storage (CCS) site, reviewed in [15]). In 
the UK, repurposing is being planned or considered for several sites, often with a focus on CCS [2]. Ultimately decommissioning strategies should be considered in light of the best current understanding and quantification of their effects on marine ecosystem functions and services.

While a growing body of literature argues that evidence-based environmental management and conservation is needed [19-23], it remains under-used, including in the marine context [24]; but see [25]. This is in spite of recent efforts to improve that evidence-base for marine management and conservation (see for instance [26-31]). In the on-going context of marine MMS decommissioning, many experts and stakeholders are advocating for an evidence-based multi-criteria approach when it comes to making decisions about decommissioning [32-37]. In the UK for example, industry operators must conduct Comparative Assessments (CA) of feasible options as part of their decommissioning proposals, which are based around five main criteria: safety, environmental, technical, societal, and economic [38, 39]. However, the extent and availability of such evidence is not yet established, which hinders multi-criteria approaches such as CAs. Due to the cross-disciplinary nature of decommissioning marine MMS, involving not only ecological considerations, but also social, political, economic and technical ones, the existing evidence appears to be dispersed across many literature sources, is in various formats, and often remains restricted within industries or government agencies. Additionally, and critically, there is also very limited direct evidence for the effects of decommissioning (for instance from pilot projects or experimental studies). The evidence used in key decision-making processes related to marine MMS (including CAs, but also licencing applications and decommissioning proposals) can lack peer-review or quantitative assessment [40], and is often based on the grey literature (notably industry-contracted research and non-peer-reviewed sources) rather than published scientific articles. In some instances, this additional published evidence base may not be available, although as recently argued in the context of coastal development, there may be conscious bias toward the use of certain literature in order to presents scenarios with a low(er) degree of environmental risk, in order to pass licensing requirements and thereby facilitate development [41]). Regardless of the reason, this may mean that a comprehensive evidence base is not considered by or fully-accessible to policy- and decision-makers, hindering revisions to conventions such as OSPAR 98/3 or OSPAR 99/13 and thus hindering effective evidencebased decision-making and practice.

To counter this limitation, several on-going projects aiming to increase this evidence base by either generating new data and information, or retrieving dispersed raw data and making them more readily accessible (such as Influence of Man-made Structures in the Ecosystem (INSITE) Programme phase 1 and 2, https://www.insit enorthsea.org). Although some studies may already be available for the effects of MMS and of their decommissioning in the sea, no comprehensive collation of this type of evidence has been undertaken by systematic review or systematic map to date (time of writing: January 2021; but see [36, 42], and a response by [43], as well as $[5,44]$ on related topics).

Here, we propose a systematic map (sensu [45]; defined further below) that will focus on published research (primarily the academic literature - see "methods" below) on the ecosystem and ecosystem services effects of MMS in the marine environment associated with either their presence or their decommissioning. We will include oil and gas infrastructures as well as OWFs, but will also consider other marine MMS that may be comparable to them in terms of their impacts (such as tidal energy installations, shipwrecks, artificial reefs, and CCS). As explained above, the term "decommission" encompasses here all possible strategies and options, from removal and disposal on shore to relocating or repurposing at sea. This evidence mapping exercise is part of the a DREAMS project (Decommissioning - Relative Effects of Alternative Management Strategies, INSITE 2 programme [https ://www.insitenorthsea.org]) which aims to develop a system to show the relative effects of implementing different decommissioning strategies in the North Sea on diverse outcomes, ranging from direct effects on ecosystem components and conservation goals to ecosystem services impacts. As far as we are aware, while some studies have reviewed the literature around the effects of MMS while in place [5, 44], no studies have systematically synthesized the published literature around their decommissioning.

Systematic maps follow a rigorous, objective, and transparent evidence synthesis methodology, and aim to collate and describe the captured evidence into a "catalogue" (see [45, 46], for further description of systematic maps). By collating and cataloguing all of the relevant and available research on the ecological and ecosystem services effects of MMS and their decommissioning in the marine environment in one place, this map will make the resultant evidence more available and accessible to all for future use. We anticipate that this will be a crucial step in the decision-making process relating to the management of marine MMS, for instance by supporting the "environmental" criterion of CAs. It will also, importantly, identify where the research gaps are, highlighting directions for future research. A wide array of elements from catalogued evidence are incorporated into systematic maps, therefore they can be extremely valuable in answering the 
variety of multi-faceted questions that researchers, stakeholders, and decision-makers may have.

As our topic has a wide scope, and is initially interested in the evidence distribution, it is better-suited for a systematic map methodology, as compared with a systematic review methodology which aims to answer a single narrower question regarding the nature of the effects (see [46] for further discussion on the differences between systematic maps and systematic reviews). The evidencebase that will be built in this mapping exercise will be used as the foundation for multiple systematic reviews and meta-analyses (where appropriate) to answer some more tightly focussed questions regarding the effects.

DREAMS is looking to map the primary literature (excluding the grey literature) not commonly used by policy- and decision-makers (see above), which will allow to determine if existing policy and decisions are based on similar or diametrically opposite evidence. By identifying and collating the available published evidence for the ecological and ecosystem service effects of marine MMS, as well as by informing the subsequent production of systematic reviews and meta-analyses on related narrower topics, and supporting the parameterisation of numerical models for alternative scenario simulations, our systematic map aims to facilitate evidence-based decision-making and the management of MMS in the marine environment.

\section{Stakeholder engagement and future work}

This protocol has been developed in consultation with the University of Plymouth, Plymouth Marine Laboratory, the Centre for Environment, Fisheries and Aquaculture Science (Cefas), and Texas A\&M University Corpus Christi. These are the partner institutions involved in DREAMS, and thus may all be considered to be stakeholders. Of note, DREAMS is supported by the INSITE programme, an industry-science collaboration.

The authors of this systematic map and its protocol represent a multi-organizational team and include members and senior representatives from each of the groups of stakeholders mentioned above. The main authors discussed and refined the scope of the map during several meetings at the initial planning stage of the project.

In addition, a small stakeholder group formed of expert representatives from academia, industry, and government agencies, provided input into this protocol, and into the evidence mapping project in general. They were contacted by emails, and telephone and video conference calls, and helped to identify key sources of literature, provided advice on technical language, and informed on decommissioning options. The list of stakeholders is listed in Additional File 6.
We expect this map to be of direct interest to each of these groups and their members/users, as well as other stakeholders across the world, in particular those in the industry and policy spheres. It is our aim that the large systematic map we will produce following this proposed protocol will be used to inform and support the creation, as part of DREAMS, of multiple systematic reviews and meta-analyses for selected topics. We envision that this map, along with the planned systematic reviews and meta-analyses that will ensue, will be useful for researchers, policy-makers, and practitioners, both in the public and private sectors to identify relevant evidence when making decisions relating to the management of manmade structures.

\section{Objective of the review Primary objectives (questions)}

The primary objectives of this systematic map are to identify and describe the evidence-base around the ecosystem and ecosystem service effects of the presence and decommissioning of MMS in the sea (i.e. during operations and after cessation of operations). We will also consider broader social and economic effects where relevant to the ecosystem and directly related to ecosystem services (e.g. fisheries displacement). This evidencebase will be built from published articles only. Here, we will not consider the grey literature, as defined by [47] and GreyNet.org (i.e. including dissertations, conference proceedings, reports, book chapters, handbooks, reports, magazine articles, newsletters, blogs, wikis, and preprints). Our decision to exclude the grey literature is based on the following reasons: (1) decommissioning decision-making, policy- and decision-makers primarily use grey literature (notably industry contracted research and also non-peer-reviewed sources) as its evidence base to inform the management of MMS but published journal article research is typically not considered. (2) The validity of grey literature as a primary evidence base has received criticism, including concerns over the degree of rigor, transparency, and accessibility, of that evidence base [40, 48]. An assessment of the evidence derived from published articles needs to be assessed and directly compared to the evidence base currently provided largely by the grey literature with respect to the effects of decommissioning of MMS.

Understanding the effects of decommissioning MMS inevitably involves understanding their effects while in place; therefore, it does not exclude studying the effects of structures that are not yet, or destined to be, decommissioned if they offer insights relevant to decommissioning. As such, all evidence pertaining to the ecosystem effects of MMS, even if outside of the decommissioning context, will be considered. This primary objective is purposely 


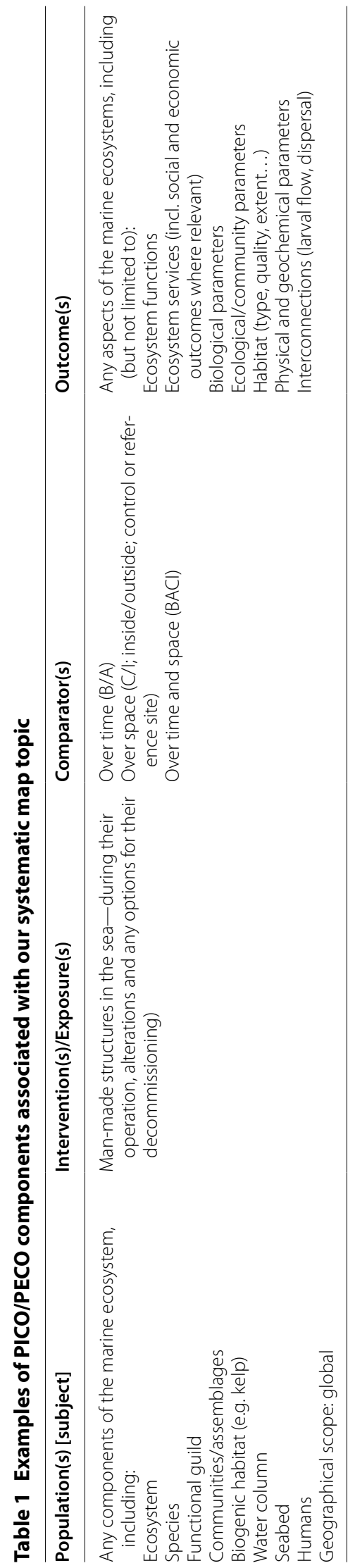


broad, incorporating multiple $\mathrm{PICO} / \mathrm{PECO}$ components (Table 1) and considering a global geographical scale so we can address narrower questions and undertake a variety of systematic reviews and geographical comparisons between the North Sea and other marine regions as the DREAMS project progresses. Our initial objectives will be to answer the following two questions:

1. What published evidence exists for the effects of marine man-made structures while in place on the marine ecosystem?

2. What published evidence exists for the effects of the decommissioning of marine man-made structures on the marine ecosystem?

\section{Secondary objectives}

Secondary objectives for this systematic map will be to answer the following questions:

3. Which decommissioning options have been 'well' studied (knowledge clusters), and which ones are lacking published evidence (knowledge gaps)?

4. What is the distribution and abundance of studies between outcomes/metrics, populations (sensu "PICO"), geographical locations, structure types/age, and years?

In other words, where are the knowledge gaps or knowledge clusters in this map relative to each of these subsets of evidence?

The outcome of our systematic map and the apparent distribution of evidence (nature, spread, trends) will inform the production of multiple systematic reviews and meta-analyses (where possible) for selected topics.

\section{Methods}

\section{Searching for articles}

A scoping exercise was conducted in the Web of Science Core Collection database (using the University of Plymouth subscription) to explore the efficiency of chosen search terms and the number of articles returned, in order to refine the search string (see Additional file 2). The final agreed search string that produced the highest efficiency was built using the sub-string presented below and returned 6133 hits as of January 26th 2021. Because our primary objective is broad and our aim is to collate the evidence about any effects of MMS on the marine environment, no specific Outcome terms were included in the search string. Instead, the search string was built using Population and Intervention terms, as well as specific terms related to the marine offshore environment and to man-made structure. Not specifying particular
Outcome terms will allow the search string to retrieve studies related to all possible outcomes. This technique has been used previously when trying to answer questions about all possible effects of interventions (e.g. [49]).

\section{Search terms}

\section{Marine and offshore qualifying terms}

$\mathrm{TS}=($ marine $\mathrm{OR}$ offshore OR pelagic OR benthic OR ocean* OR sea OR shelf OR shelves OR coast*).

AND

\section{Population terms}

TS $=($ ecosystem $\$$ OR habitat\$ OR seabed\$ OR sediment\$ OR "ecological system" benthos OR environment* OR species OR assemblage\$ OR communit* OR population\$ OR fisher* OR service\$ OR human\$ OR people).

AND

\section{Intervention and exposure terms}

$\mathrm{TS}=\left(\right.$ plac $^{*}$ OR install* OR deploy* OR decommission* OR manag* OR reefing OR toppling OR topping OR repurpose* OR relocat* OR alter* OR salvag* OR remov*) AND.

\section{Man-made structure terms}

$\mathrm{TS}=$ ("man-made structure\$" OR "offshore structure\$" OR "artificial structure\$" OR "oil and gas" OR "oil and gaz" OR "oil \& gas" OR "oil \& gaz" OR "oil rig\$” OR "petroleum installation" OR "windfarm\$" OR "wind farm\$" OR "wind turbine*" OR MREI OR "wave farm*" OR "tidal energy" OR "tidal stream*" OR "artificial reef\$" OR wreck\$ OR "shipwreck\$” OR CCS OR "carbon capture" OR "carbon storage").

\section{Sources of literature to be searched Databases, repositories, and organisational websites}

We will search the literature for relevant articles, using the search string detailed above entered into several publication databases, search engines, repositories, and organisational databases and websites (Table 2). Where searches cannot be performed using the agreed search string or a modified syntax of the search string - for instance on some organisational websites - manual systematic hand-searches will be performed and publications that pass the first stage of screening (at title and abstract level) will be included. Searches will be undertaken in English initially, for time constraint, but also because it is likely that the majority of the relevant literature (pertaining to offshore structures and industries) is published in English. If time allows, searches will also be undertaken in French (language spoken by the review team and relevant to the North-Sea context). 
Table 2 List of literature sources that will be systematically searched for relevant studies

\begin{tabular}{|c|c|}
\hline Priority sources to search & Secondary sources to search \\
\hline Web of Science Core Collection ${ }^{\mathrm{ab}}$ & Wageningen University \& Research repository ${ }^{c}$ \\
\hline Scopus ${ }^{\mathrm{b}}$ & Royal Belgian Institute of Natural Science repository ${ }^{c}$ \\
\hline $\begin{array}{l}\text { Databases within the Aquatic Sciences and Fisheries Abstracts pub- } \\
\text { lisher platform ProQuest }{ }^{b}\end{array}$ & Alfred Wegener Institute repository ${ }^{c}$ \\
\hline Collaboration for Environmental Evidence libraryc & Royal Netherlands Institute of Sea Research repository ${ }^{c}$ \\
\hline INSITE database and website & $\begin{array}{l}\text { Collaborative Offshore Wind Research Into The Environment database (COW- } \\
\text { RIE_-publications only) }\end{array}$ \\
\hline Environmental Studies Program Information System $\left(\right.$ ESPIS) ${ }^{c}$ & Department for Environment, Food and Rural Affairs ${ }^{d}$ \\
\hline \multirow{13}{*}{ 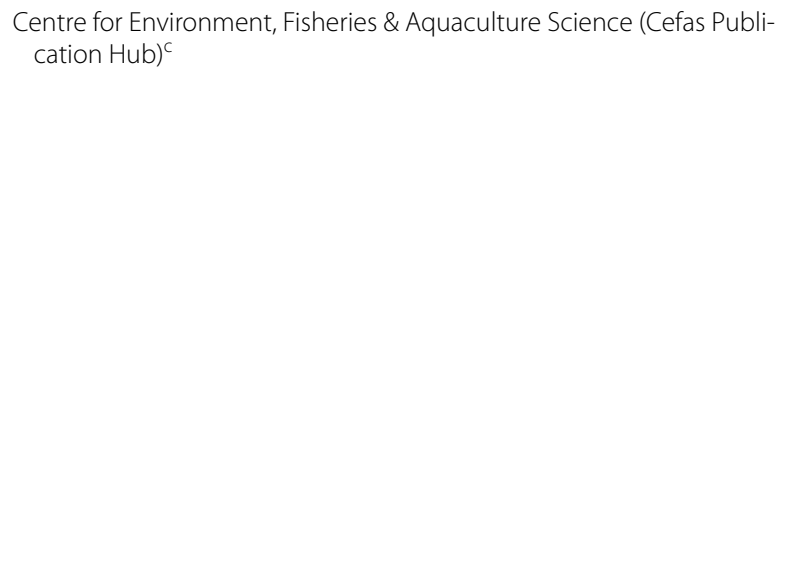 } & Joint Nature Conservation Committee ${ }^{d}$ \\
\hline & Marine Scotland ${ }^{d}$ \\
\hline & Natural Resources Wales - Marine and coastal evidence reports ${ }^{c}$ \\
\hline & English Nature Access to Evidence ${ }^{d}$ \\
\hline & Environment Agency ${ }^{d}$ \\
\hline & Marine Management Organisation ${ }^{d}$ \\
\hline & International Council for the Exploration of the Sea ${ }^{d}$ \\
\hline & Helsinki Convention (HELCOM) ${ }^{c}$ \\
\hline & Oslo-Paris Commission (OSPAR) ${ }^{c}$ \\
\hline & Applied Ecology Resources ${ }^{c}$ \\
\hline & Nature-based Solutions Initiative evidence platform \\
\hline & BSH (Federal Maritime and Hydrographic Agency) \\
\hline & Offshore Petroleum Regulator for Environment and Decommissioning (OPRED) \\
\hline
\end{tabular}

a WoS was searched using the University of Plymouth subscription, citation indices are listed in Additional file 2

b Sources searched using the agreed search string (or a variant thereof)

c Sources where our search string could not be entered and a manual hand-search was performed

${ }^{d}$ Where institutions were contacted directly to ask for their relevant publications

Due to time constraints, literature sources will be split into "priority sources" and "secondary sources". Searches, screening, and meta-data extraction will be performed first on the studies returned from the "priority sources", followed if time allows by studies returned from the "secondary sources" (minus duplicates).

\section{Other searches (snowballing)}

Where a systematic review or a meta-analysis is found and passes the screening process, all studies reviewed within it will be included, as well as the meta-analysis. The systematic review itself will be included only if it contains new primary data. Where a verbose (non-systematic) review is found, all relevant studies referenced within it will be subjected to the screening process and included if they pass it, but the review itself will not be, unless it also provides new or collective data. Other publications that pass the screening process and will be included in the systematic map will not be scanned for relevant citations due to time restrictions.
Google Scholar will be queried to supplement our searches, and the first 200 returns selected, following recommendations by [50].

\section{Assembling a library of search results}

Publications retrieved following our searches will be entered into a single library in the reference manager Zotero. Duplications will be removed prior to the screening process.

\section{Comprehensiveness of the searches}

A list of 25 benchmark publications was compiled by the members of the review team based on their own expertise, previous systematic maps and reviews, and general searches on the internet (see Additional file 2). Searches were modified and refined until $\geq 75 \%$ of the benchmark publications were retrieved.

\section{Article Screening and Study eligibility criteria Screening process}

We will screen the publications in two stages: (1) at title and abstract level, and (2) at full text level. At each 
stage, we will decide whether to include a publication or exclude it from the map, based on agreed eligibility criteria (see below and decision trees in Additional file 3). Where the relevance of a publication is unclear at stage 1 , it will be included and assessed at stage 2, to err on the side of inclusion. We will record the number of publications included/excluded at each stage. Records will be kept of all the publications excluded at stage 2 (full text), together with the reasons for their exclusion. Similarly, records will be kept of publications that could not be located or accessed. Where a publication cannot be located or accessed in full using the University of Plymouth subscription, we will attempt to contact the authors with a request for the full text. These records will be made available.

A team of nine independent reviewers will participate in the screening process. To ensure consistency and accuracy throughout the screening process amongst the members of the review team, a random subset of 100 publications will be independently screened by all reviewers. Results will be analysed using Cohen's Kappa test (Cohen 1960) and used to ascertain the level of agreement amongst the reviewers. If the initial results show 'substantial' $(\mathrm{K}=0.61-0.8)$ or 'almost perfect' agreement $(\mathrm{K}=0.81-1.0)$, the reviewers will not receive further training, but disagreements will be discussed nonetheless to ensure the best possible outcomes are achieved. If the Kappa score is less than 0.61, disagreements will be discussed and resolved, and Kappa scores will be calculated for another set of 50 publications minimum. This process will be repeated until the Kappa score is greater than 0.61 .

In the instance that a retrieved publication was authored or co-authored by one or more members of the review team, the publication will be referred to another reviewer for assessment.

\section{Eligibility criteria}

Publications meeting the following criteria will be included in our map (see also Table 1):

\section{Relevant population/subject}

All relevant marine components, including ecosystems, populations, species, communities, assemblages, as well as the water column, habitats, sediments and the seabed will be considered. Additionally, we will consider humans as a population, for instance for evidence related to ecosystem services. While our priority question as part of DREAMS pertains to MMS in the North Sea, the geographical scope considered will be global (i.e.: evidence from all marine environments globally) to allow us to draw comparisons between geographical locations.

\section{Relevant interventions/exposures}

We aim to include all man-made structures that are present in the marine environment and that may be comparable to oil and gas infrastructure in terms of their impacts, in order to compare the effects of different types, intended purposes and decommissioning options. These will include: oil and gas structures, OWF, tidal energy installations and other marine renewable energy installations (MREI), shipwrecks, artificial reefs, and CCS. However, we realise considering such a wide range of subjects may not be feasible in the time allocated to this project. Therefore, we will prioritise extraction of meta-data for studies investigating the types of structures that are currently being, or soon to be, decommissioned: (1) oil and gas structures (rigs, platforms, jackets, pipelines, stabilising material) and (2) wind farms. We will then extract the meta-data from studies of other types of MMS, to allow for comparisons, in the following order of priority: (3) artificial reefs, (4) shipwrecks, (5) tidal energy installations and other MREIs, and (6) CCS.

\section{Relevant comparators}

Ideally, all studies would include suitable comparators. Those include temporal comparators (before/after, time series), spatial comparators (between different structures or sites, near/inside vs far/outside a structure or site, as well as depth comparisons), as well as procedural controls and reference sites (Control/Impact). For instance, a study could compare one specific intervention/exposure against another, such as comparing the outcomes of different decommissioning strategies. Nevertheless, we acknowledge that no suitable comparators may be possible in the marine context, and that oftentimes relevant studies may not include one. As such we will also consider studies that did not include a comparator.

\section{Relevant outcomes/metrics}

We will focus on studies that assess any ecosystem effects on any components of the marine environment. This will include (but is not limited to) ecological and biological effects (such as ecosystem function, ecosystem structure, community/assemblage composition, biological diversity, species presence/absence/abundance/biomass, habitat type and quality etc.); physical and geochemical effects (such as grain size, sediment type, flow); effects on connectivity (e.g. propagule and larval dispersal; population connectivity); as well as ecosystem service effects and social and economic effects where relevant and directly related to an ecosystem service outcome (such as fisheries displacement) (see Additional file 4 for the list of 
anticipated relevant outcomes/metrics categories). If we find additional relevant outcomes/metrics for these populations/subjects during the meta-data extraction process, these will be added to the list.

\section{Relevant study designs}

We will include evidence from studies that have experimentally tested, measured or assessed effects, as well as observational studies that have recorded or quantified effects or relevant outcomes. We will also include systematic reviews and meta-analyses. We will not include modelling studies, purely theoretical or conceptual studies, nor will we include verbose reviews, unless they provide or are based upon new empirical quantified effects.

\section{Study validity assessment}

For the purpose of this systematic map, the validity of each piece of evidence will not be assessed or weighed. However, information regarding the design of each study will be coded, and thus can be considered by the users of the map when interpreting the evidence. The validity of relevant studies will be assessed during the production of the planned systematic reviews and meta-analyses.

\section{Data coding strategy}

For the purpose of our systematic map, the following meta-data (information describing each study) will be extracted and coded for each eligible study described in each publication that passes stage 2 of the screening process following a standardised coding framework (see Additional file 4 for the full framework, and Additional file 5 for an example). Note that a single publication can describe more than one study. A database will be built that will contain all included studies and their coded meta-data. Each study will be given a unique identifier. We will code (1) PICO/PECO components (as described above in "Eligibility criteria" and Table 1); these are information related to the study, such as study year and duration, study site (at two or more geographical levels), structure identity and type (at two or more hierarchical levels), study design, intervention/exposure (at two or more category levels), outcome, and population (at two or more category levels). (2) We will also code bibliographic information related to the publication (article reference; article year of publication; journal/report name). Finally, we will code (3) reviewer information (which team member extracted the data). When necessary information is missing or unclear, we will clearly state it by coding the associated fields with the term "unspecified" or "unclear" respectively. In the instance where more than one publication presents the same study outcome or data, both will be kept as part of this map, but a note will be made. For subsequent statistical and counting purposes (e.g. visualisation of evidence distribution, see "Study mapping and presentation" section below), only one of the duplicated study will be retained.

To ensure the consistency and accuracy of data extraction and validity of data coding, the meta-data from an initial 10 publications (selected at random following stage 1 screening) will be extracted and coded by all members of the review team. Kappa scores will be calculated to test agreement between reviewers on selected meta-data (e.g. agreement about which interventions and structure types were studied). Disagreements will be discussed, and the eligibility criteria will be revised accordingly to improve the efficacy of the process. If the Kappa score is less than 0.61 'substantial agreement' (as above), then another 10 publications will be coded and compared again. This process will be repeated until the Kappa score is greater than 0.61.

\section{Study mapping and presentation}

The evidence will be presented as a queryable database (which could be also described as a catalogue of studies, interventions, species, effects, etc.), and analysed in a variety of ways, for instance by intervention type, by effect type, by geographic location, by structure type, or by year. This systematic map, or "catalogue", will ultimately be freely accessible online. The results will be visually presented as part of an open-access manuscript presenting the systematic map, to show knowledge gaps and knowledge clusters using various figure formats. For instance it will include the production of geographical maps of evidence spread to show geographical knowledge gaps and knowledge clusters.

Based on these results, we will make recommendations on priorities for future research related to the management and decommissioning of marine MMS. As mentioned above, these results will also inform subsequent systematic reviews and meta-analyses on related narrow questions pertaining to the effects of marine MMS and their decommissioning. Examples of a-priori planned systematic review questions include "What are the effects of different types of marine MMS on emergent epifaunal communities", and "What are the effects of repurposing an MMS into an AR on marine biodiversity/fish population/ species abundance/composition".

\section{Supplementary Information}

The online version contains supplementary material available at https://doi. org/10.1186/s13750-021-00218-y.

Additional file 1: Authors' declaration and checklist of adherence to the ROSES guidelines. 
Additional file 2: Building and testing the search string against a list of benchmark publications.

Additional file 3: Decision trees for screen 1 and screen 2.

Additional file 4: Working framework for meta-data coding

Additional file 5: Example of the meta-data extracted from a relevant study and coded using the chosen fields.

Additional file 6: List of stakeholders involved in the project and who advised on the map.

\section{Acknowledgements}

The authors acknowledge the financial and technical support provided by NERC and the INSITE programme. We are grateful to all the members of our stakeholder group for their contribution and willingness to advise on this project. All stakeholders named in Additional file 6 gave their consent to have their names and/or affiliations disclosed. We thanks the two anonymous reviewers as well as the editor for their comments and suggestions during the peer-review process.

\section{Authors' contributions}

AJL wrote the protocol, with revisions from AMK, AMQ, CP, GL, MS, MT, NB and PJS. AJL conceived the literature search strategy following discussions with all co-authors. AJL, AMK, CP, GL, LM, MS, MT, NB and PJS contributed to the initial discussion and integration of the systematic map methods into the DREAMS project. All authors read and approved the final manuscript.

\section{Funding}

This map will be undertaken within the agreed work of DREAMS, which is financed by the UK Natural Environment Research Council, Grants No. NE/ T010843/1 and NE/T010835/1.

\section{Availability of data and materials}

Data sharing is not applicable to the publication of this systematic map protocol as no datasets were generated.

\section{Ethics approval and consent to participate}

Not applicable.

\section{Consent for publication}

Not applicable.

\section{Competing interests}

The authors declare having no competing interests.

\section{Author details}

${ }^{1}$ School of Biological and Marine Sciences, University of Plymouth, Drake Circus, Plymouth PL4 8AA, UK. ${ }^{2}$ Cefas - Centre for Environment, Fisheries and Aquaculture Science, Lowestoft Laboratory, Pakefield Road, Lowestoft, Suffolk NR33 OHT, UK. ${ }^{3}$ PML - Plymouth Marine Laboratory Prospect Place, The Hoe, Plymouth PL13DH, UK.

Received: 7 December 2020 Accepted: 28 January 2021

Published online: 12 February 2021

\section{References}

1. Bugnot AB, Mayer-Pinto M, Airoldi L, Heery EC, Johnston EL, Critchley LP, et al. Current and projected global extent of marine built structures. Nat Sustain. 2020;1-9.

2. OGUK. Decommissioning Insight Report. 2020. https://cld.bz/1 kyzb 6w/6/. Accessed 7 Jan 2021

3. Parente V, Ferreira D, dos Santos EM, Luczynski E. Offshore decommissioning issues: Deductibility and transferability. Energy Policy. 2006:34(15):1992-2001.

4. Fowler AM, Jørgensen A-M, Svendsen JC, Macreadie PI, Jones DOB, Boon $A R$, et al. Environmental benefits of leaving offshore infrastructure in the ocean. Front Ecol Environ. 2018;16(10):571-8.
5. Ashley MC, Mangi SC, Rodwell LD. The potential of offshore windfarms to act as marine protected areas - A systematic review of current evidence. Mar Policy. 2014:45:301-9.

6. Topham E, Gonzalez E, McMillan D, João E. Challenges of decommissioning offshore wind farms: Overview of the European experience. In: Journal of Physics: Conference Series. 2019. https://www.scopus.com/inward/ record.uri?eid $=2-s 2.0-85066449302 \&$ doi $=10.1088 \% 2 f 1742-6596 \% 2 f 122$ 2\%2f1\%2f012035\&partnerlD=40\&md5=eba7cd9b0eaa03c05fa77d6c5 ec4e491

7. Topham E, McMillan D. Sustainable decommissioning of an offshore wind farm. Renew Energy. 2017;102:470-80.

8. Dannheim J, Bergstrom L, Birchenough SNR, Brzana R, Boon AR, Coolen JWP, et al. Benthic effects of offshore renewables: identification of knowledge gaps and urgently needed research. Ices J Mar Sci. 2020;77(3):1092-108.

9. Knights AM, Culhane F, Hussain SS, Papadopoulou K-N, Piet GJ, Raakær J, et al. A step-wise process of decision-making under uncertainty when implementing environmental policy. Environ Sci Policy. 2014;39:56-64

10. Ounanian K, van Tatenhove JPM, Ramirez-Monsalve P. Midnight at the oasis: does restoration change the rigs-to-reefs debate in the North Sea? J Environ Policy Plan. 2020;22(2):211-25.

11. Piet $\mathrm{GJ}$, Jongbloed $\mathrm{RH}$, Knights $\mathrm{AM}$, Tamis JE, Paijmans $\mathrm{AJ}$, van der Sluis MT, et al. Evaluation of ecosystem-based marine management strategies based on risk assessment. Biol Conserv. 2015;186:158-66.

12. Piet GJ, Knights AM, Jongbloed RH, Tamis JE, de Vries P, Robinson LA. Ecological risk assessments to guide decision-making: Methodology matters. Environ Sci Policy. 2017:68:1-9.

13. Jørgensen D. OSPAR's exclusion of rigs-to-reefs in the North Sea. Ocean Coast Manag. 2012:58:57-61.

14. Fowler AM, Jorgensen A-M, Coolen JWP, Jones DOB, Svendsen JC, Brabant $\mathrm{R}$, et al. The ecology of infrastructure decommissioning in the North Sea: what we need to know and how to achieve it. Ices J Mar Sci. 2020;77(3):1109-26.

15. Sommer B, Fowler AM, Macreadie PI, Palandro DA, Aziz AC, Booth DJ. Decommissioning of offshore oil and gas structures - Environmental opportunities and challenges. Sci Total Environ. 2019;658:973-81.

16. Techera EJ, Chandler J. Offshore installations, decommissioning and artificial reefs: Do current legal frameworks best serve the marine environment? Mar Policy. 2015;59:53-60.

17. Bull AS, Love MS. Worldwide oil and gas platform decommissioning: a review of practices and reefing options. Ocean Coast Manag. 2019:168:274-306.

18. Kaiser MJ, Pulsipher AG. Rigs-to-reef programs in the Gulf of Mexico. Ocean Dev Int Law. 2005:36(2):119-34.

19. Dicks LV, Hodge I, Randall NP, Scharlemann JP, Siriwardena GM, Smith $\mathrm{HG}$, et al. A transparent process for "evidence-informed" policy making. Conserv Lett. 2014;7(2):119-25.

20. Keene M, Pullin AS. Realizing an effectiveness revolution in environmental management. J Environ Manage. 2011;92(9):2130-5.

21. Pullin AS, Knight TM. Support for decision making in conservation practice: an evidence-based approach. J Nat Conserv. 2003;11(2):83-90.

22. Sutherland WJ, Pullin AS, Dolman PM, Knight TM. The need for evidencebased conservation. Trends Ecol Evol. 2004;19(6):305-8.

23. Sutherland WJ, Taylor NG, MacFarlane D, Amano T, Christie AP, Dicks $\mathrm{LV}$, et al. Building a tool to overcome barriers in research-implementation spaces: The Conservation Evidence database. Biol Conserv. 2019;238:108199.

24. Cvitanovic C, Fulton CJ, Wilson SK, van Kerkhoff L, Cripps IL, Muthiga N. Utility of primary scientific literature to environmental managers: an international case study on coral-dominated marine protected areas. Ocean Coast Manag. 2014;102:72-8.

25. MacLean SA, Rooper CN, Sigler MF. Corals, canyons, and conservation: science based fisheries management decisions in the Eastern Bering Sea. Front Mar Sci. 2017:4:142

26. Boström-Einarsson L, Babcock RC, Bayraktarov E, Ceccarelli D, Cook N, Ferse SC, et al. Coral restoration-A systematic review of current methods, successes, failures and future directions. PLoS ONE. 2020;15(1):e0226631.

27. Brooks WR, Rudd ME, Cheng SH, Silliman BR, Gill DA, Ahmadia GN, et al. Social and ecological outcomes of conservation interventions in tropical coastal marine ecosystems: a systematic map protocol. Environ Evid. $2020 \cdot 9(1) \cdot 9$ 
28. d'Armengol L, Prieto Castillo M, Ruiz-Mallén I, Corbera E. A systematic review of co-managed small-scale fisheries: Social diversity and adaptive management improve outcomes. Glob Environ Change. 2018;52:212-25.

29. Gallacher J, Simmonds N, Fellowes H, Brown N, Gill N, Clark W, et al. Evaluating the success of a marine protected area: A systematic review approach. J Environ Manage. 2016;183:280-93.

30. Lemasson A, Pettit L, Smith R, Sutherland W. Subtidal Benthic Invertebrate Conservation: Global Evidence for the Effects of Interventions. 2020.

31. Mizrahi M, Diedrich A, Weeks R, Pressey RL. A systematic review of the socioeconomic factors that influence how marine protected areas impact on ecosystems and livelihoods. Soc Nat Resour. 2019;32(1):4-20.

32. Birchenough SNR, Degraer S. Science in support of ecologically sound decommissioning strategies for offshore man-made structures: taking stock of current knowledge and considering future challenges. Ices J Mar Sci. 2020;77(3):1075-8.

33. Burdon D, Barnard S, Boyes SJ, Elliott M. Oil and gas infrastructure decommissioning in marine protected areas: System complexity, analysis and challenges. Mar Pollut Bull. 2018;135:739-58.

34. Fortune IS, Paterson DM. Ecological best practice in decommissioning: a review of scientific research. Ices J Mar Sci. 2020;77(3):1079-91.

35. Fowler AM, Macreadie PI, Jones DOB, Booth DJ. A multi-criteria decision approach to decommissioning of offshore oil and gas infrastructure. Ocean Coast Manag. 2014;87:20-9.

36. Mangano MC, Sara G. Collating science-based evidence to inform public opinion on the environmental effects of marine drilling platforms in the Mediterranean Sea. J Environ Manage. 2017;188:195-202.

37. Martins ID, Moraes FF, Távora G, Soares HLF, Infante CE, Arruda EF, et al. A review of the multicriteria decision analysis applied to oil and gas decommissioning problems. Ocean Coast Manag. 2020;184:105000.

38. Oil \& Gas UK. . Guidelines for Comparative Assessment in Decommissioning Programmes. The UK Oil and Gas Industry Association Limited: London; 2015.

39. OPRED Offshore Petroleum Regulator for Environment and Decommissioning. Oil and gas: decommissioning of offshore installations and pipelines. 2018. https://www.gov.uk/guidance/oil-and-gas-decommissi oning-of-offshore-installations-and-pipelines
40. Linkov I, Loney D, Cormier S, Satterstrom FK, Bridges T. Weight-of-evidence evaluation in environmental assessment: review of qualitative and quantitative approaches. Sci Total Environ. 2009;407(19):5199-205.

41. Firth LB, Airoldi L, Bulleri F, Challinor S, Chee S-Y, Evans AJ, et al. Greening of grey infrastructure should not be used as a Trojan horse to facilitate coastal development. J App Ecol. 2020;57(9):1762-8.

42. Mangano MC, Sarà G. The author's reply to N.R. Haddaway. J Environ Manage. 2017;197:114-6.

43. Haddaway NR. Response to "Collating science-based evidence to inform public opinion on the environmental effects of marine drilling platforms in the Mediterranean Sea." J Environ Manage. 2017;203:612-4.

44. Methratta ET, Dardick WR. Meta-Analysis of Finfish Abundance at Offshore Wind Farms. Rev Fish Sci Aquac. 2019;27(2):242-60.

45. James KL, Randall NP, Haddaway NR. A methodology for systematic mapping in environmental sciences. Environ Evid. 2016;5(1):7.

46. Collaboration for Environmental Evidence. Guidelines and Standards for Evidence synthesis in Environmental Management. Version 5.0. AS Pullin, GK Frampton, B Livoreil \& G Petrokofsky, Eds; 2018. www.environmentalev idence.org/information-for-authors.

47. Mahood Q, Van Eerd D, Irvin E. Searching for grey literature for systematic reviews: challenges and benefits. Res Synth Methods. 2014;5(3):221-34.

48. McAuley L, Tugwell P, Moher D. Does the inclusion of grey literature influence estimates of intervention effectiveness reported in meta-analyses? Lancet. 2000;356(9237):1228-31.

49. Ouédraogo D-Y, Sordello R, Brugneaux S, Burga K, Calvayrac C, Castelin $M$, et al. What evidence exists on the impacts of chemicals arising from human activity on tropical reef-building corals? A systematic map protocol. Environ Evid. 2020;9(1):18.

50. Haddaway NR, Collins AM, Coughlin D, Kirk S. The role of Google Scholar in evidence reviews and its applicability to grey literature searching. PLoS ONE. 2015;10(9):e0138237.

\section{Publisher's Note}

Springer Nature remains neutral with regard to jurisdictional claims in published maps and institutional affiliations.
Ready to submit your research? Choose BMC and benefit from:

- fast, convenient online submission

- thorough peer review by experienced researchers in your field

- rapid publication on acceptance

- support for research data, including large and complex data types

- gold Open Access which fosters wider collaboration and increased citations

- maximum visibility for your research: over 100M website views per year

At BMC, research is always in progress.

Learn more biomedcentral.com/submissions 